\title{
A new dissection procedure for learning of echocardiography
}

\author{
Hiromi Suenaga* \\ Faculty of Health Sciences, Yamaguchi University School of Medicine, Ube, Yamaguchi 755-8505, Japan
}

\begin{abstract}
Ultrasonography has been an indispensable diagnostic technique for a significant period of time. A dissection understanding of each target area of the body is essential when applying ultrasonography. However, specialized dissection procedures for ultrasonography are rarely presented. With this in mind we devised a new method for learning echocardiography for novice students.

We developed a new learning program to assist students to learn echocardiography. This involves them dissecting pig hearts in a way that mirrors $3 \mathrm{D}$ echocardiographic images, and involves seven steps. We anticipate that this dissection procedure will be an effective learning program in ultrasound education. The dissection methods will lead to greater understanding of the $3 \mathrm{D}$ structure of the human heart.
\end{abstract}

\section{Introduction}

Diagnostic imaging using ultrasound equipment is an essential modality in clinical practice that provides several advantages, including non-invasiveness, safety, and the ability to acquire real-time information. For newcomers to ultrasound imaging, an important aspect of learning ultrasound technology is an anatomical understanding of each organ. However, understanding organ anatomy and 3D structure is not easy even for clinicians and ultra-sonographers. With recent advances in ultrasound equipment and with the advent of real-time 3D images, the familiarity with the $3 \mathrm{D}$ structure of an organ are needed for more strongly than ever before for understanding of the new ultrasound imaging. The precise 3D structure of the components of the heart can be especially difficult to understand. To date, many atlas, schemes, models, and gross dissection have been widely used to teach cardinal cardiac anatomy to students. However, these teaching materials are not effective in the acquisition of knowledge of cross-sectional anatomy required to understand echocardiography . To enhance education in ultrasound diagnostic technology, this study established a new heart dissection procedure with the use of a pig heart appropriate for learning about echocardiography.

\section{Heart dissection procedure}

1. A pig heart, the scalpel and a pinch for dissection are prepared. With the heart in frontal view, a line is made connecting the cardiac apex with the position of the right coronary cusp of the aortic valve. This corresponds to the chest wall side of a parasternal long-axis view on echocardiography (Figure 1-A).

2. Mark a point corresponding to $1 / 4$ of the height from the cardiac base along line 1 and the height of the tricuspid valve annulus. This is the most inferior point on the chest wall side on a short-axis view at the aortic valve level (Figure 1-A).

3. Dissect obliquely upward along a line from point 2 connecting the area just above the aortic valve, the left atrium, and the right atrium. Do not dissect the atrial septum. This section at the cardiac base corresponds to the aortic valve level on echocardiography (Figure 1-A).
4. The cardiac apex side of the section is observed from the cardiac base side. The aortic valve on the chest wall side is the right coronary cusp. Mark a line that connects line 1, the right coronary cusp, and the points of maximum diameter of the left ventricle (crossing the center of the anterior mitral valve leaflet and the posterior mitral valve leaflet (Figure 1-B).

5. Dissect along line 4 in order of the anterior wall of the right atrium, the aorta, the ventricular septum, and the anterior mitral valve leaflet. Do not dissect the posterior wall of the left ventricle. The section on the left side facing from the front corresponds to a parasternal long-axis view on echocardiography (Figure 1-C, D).

6. Open the section 5 and dissect along a line connecting the most developed parts of the anterior papillary muscle and the posterior papillary muscle in the left ventricle. This section near the cardiac base corresponds to a short-axis view at the papillary muscle level on echocardiography (Figure 1-D, E).

7. From the section, as in 6 , dissect along a line connecting the cusps of the anterior mitral valve leaflet and the posterior mitral valve leaflet. Similarly, this section near the cardiac base corresponds to a shortaxis view at the mitral valve level on echocardiography (Figure 1-F).

\section{Discussion}

With recent advances in ultrasound equipment and with the advent of real-time $3 \mathrm{D}$ images, the familiarity with the $3 \mathrm{D}$ structure of an organ are needed for more strongly than ever before for understanding

Correspondence to: Hiromi Suenaga, Ph.D, Faculty of Health Sciences, Yamaguchi University Graduate School of Medicine, 1-1-1 Minamikogushi, Ube, Yamaguchi 755-8505, Japan, Tel: +81 83622 2133; Fax: +81 83622 2133; E-mail: hiro1204@yamaguchi-u.ac.jp

Key words: ultrasonography, echocardiography, dissection procedure

Received: July 04, 2017; Accepted: July 26, 2017; Published: July 28, 2017 


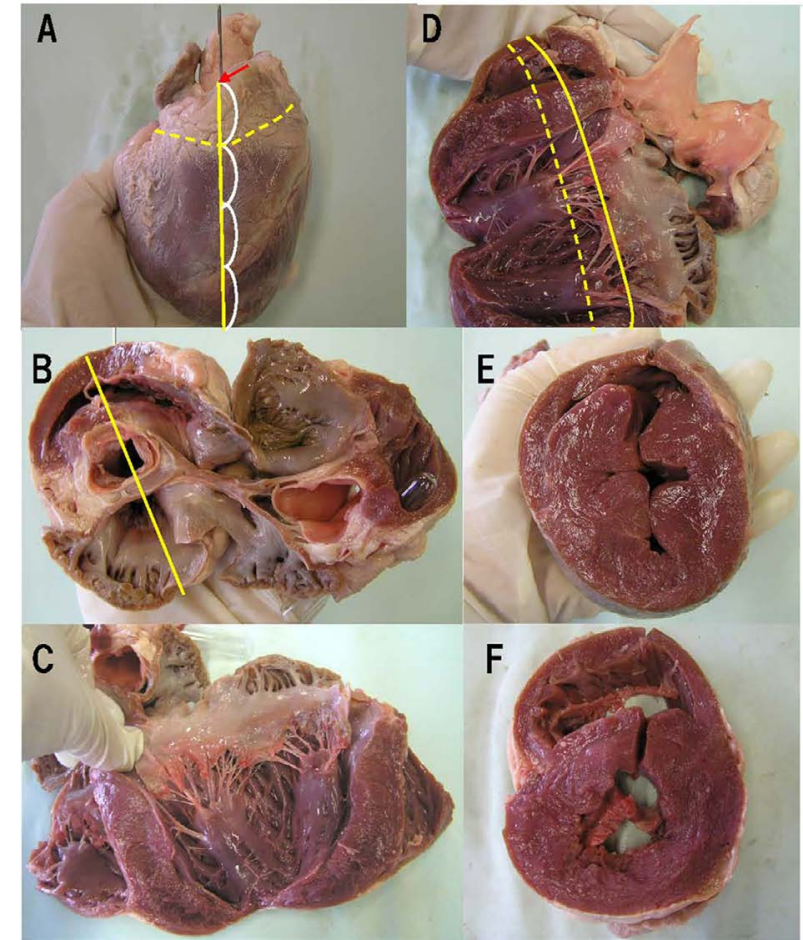

Figure 1. A. Heart, frontal view (continuous line: parasternal long-axis view; chest wall side, interrupted line: short-axis view: aortic valve level, arrow: Aortic valve: right coronary cusp level) B: Heart exposed (short-axis view; aortic valve level, line: parasternal long-axis view) C: Heart exposed (parasternal long-axis view) D: Heart exposed (parasternal longaxis view, continuous line: mitral valve level, interrupted line: papillary muscle level) E: Short-axis view; papillary muscle level (R: right side, L; left side) F: Short-axis view; mitra valve level (R: right side, $\mathrm{L}$; left side)
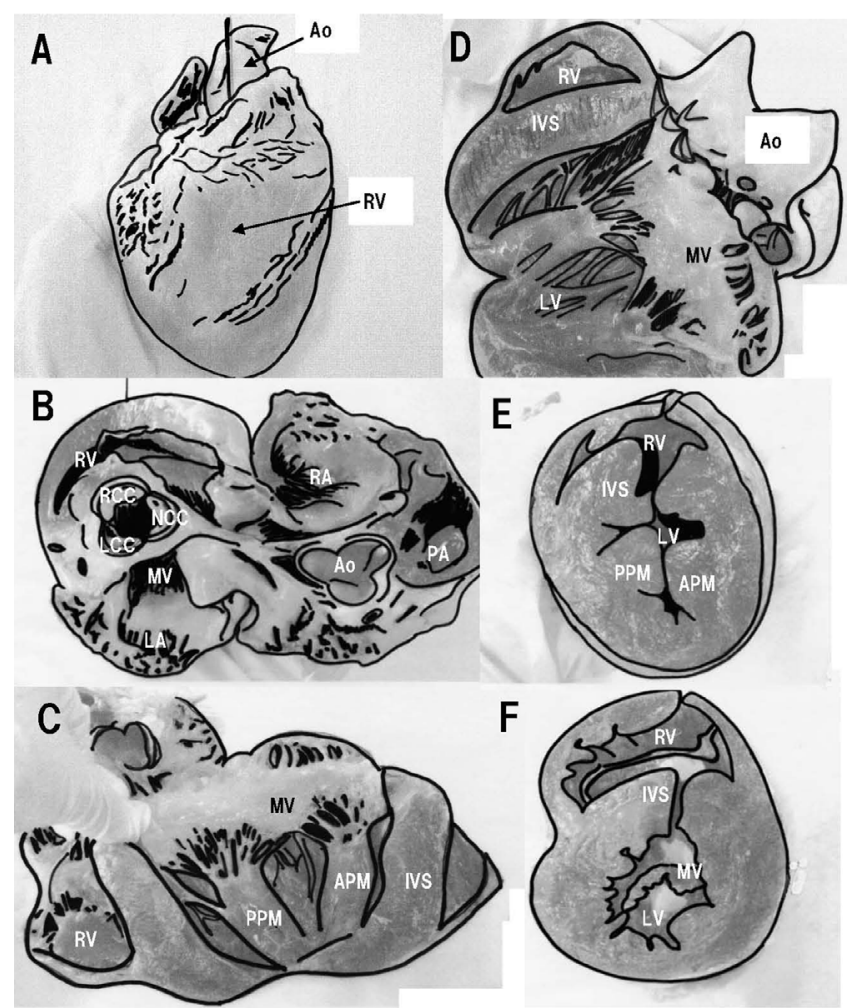

Figure 2. $\square$ The scheme of Fig. 1 (Ao: Aorta, RV: right ventricle, RA: right atrium, LV: left ventricle, LA: left atrium, PA: pulmonary artery, RCC: right coronary cusp, NCC: noncoronary cusp, LCC: left coronary cusp, MV: mitral valve, APM: anterior papillary muscle, PPM: posterior papillary muscle, IVS: inter-ventricular septum) of the new ultrasound imaging. Previous studies about teaching cardiac anatomy reported educational applications of computed tomography [1], a computer program called the Virtual Heart from UC Davis [2] and the clay models [3]. However, two former are less experience in actually seeing, touching and learning by hands, the latter also do not adequately delineating the subtleties of cardiac anatomy. To date, dissection methods to help understand various structures and tissues have included external dissection of the left ventricle, right ventricle, left atrium, and right atrium, to observe internal structure, or a division into parts of the lower heart, including the left ventricle and right ventricle, and upper heart, including left atrium and right atrium, to observe and understand their structure. However, to echocardiography tomographic images, these are not rational methods to understand the $3 \mathrm{D}$ anatomy of cardiac structures that exist in the order of the direction of an ultrasound beam. Therefore, it seemed beneficial to find a specialized new dissection procedure for learning echocardiography. The dissection procedure that we devised makes it easy to understand, when dissecting the heart from externally in a particular direction, which major echocardiography tomographic images would be acquired, and by dissecting in order of the direction of an ultrasound beam, the 3D structure on echocardiography tomographic images is more easily understood. In addition, because the heart is not divided into parts, and the cardiac appendages are not destroyed, even after proceeding to a certain step in dissection, one can return to the previous step to review cardiac structure. Therefore, a single pig heart can be used more effectively. We anticipate that this new dissection procedure will be an effective learning program in ultrasound education.

\section{Conflict of interest}

None

\section{Ethical considerations}

This article does not contain any studies with human or animal subjects performed by the any of the authors.

\section{References}

1. Shapiro LB, Watt-Smith SR, Milosevic AM, Walters ID, Young P, et al. (1998) Crosssectional imaging of a cadaveric human heart. Clin Anat 11: 75-80. [Crossref]

2. Bailey P (1997) Virtual vet-med realities. High school and vet students click into their subject matter. Dateline UC Davis Faculty and Staff newspaper.

3. Oh CS, Kim JY, Choe YH (2009) Learning of Cross-Sectional Anatomy Using Clay Models. Anat Sci Educ 2: 156-159. [Crossref]

Copyright: (C2017 Suenaga H. This is an open-access article distributed under the terms of the Creative Commons Attribution License, which permits unrestricted use, distribution, and reproduction in any medium, provided the original author and source are credited. 